\title{
To Numerical Solution of Singular Perturbed Equations Transformed to the Best Argument*
}

\author{
E.B. Kuznetsov and S.D. Krasnikov \\ Moscow Aviation Institute, Volokolamskoe shosse 4, \\ 125993, Moscow, Russia \\ Kuznetsov@mai.ru, \\ sergeykr@mtu-net.ru
}

\begin{abstract}
We consider the numerical solution of initial value problem for the system of ordinary differential singular perturbed equations. The integral curve of the problem is constructed using method of continuation with respect to a parameter. We can choose the best parameter in any step of integration process. It is found that the best argument of the Cauchy problem is the arc length of the integral curve of the problem. Transformed to the best argument Cauchy problem has a number advantages in comparison with the Cauchy problem over the usual statement [1]. The right - hand side of each transformed equation does not exceed unit. Moreover, the squared norm of the system right - hand sides is always equal to unit. Also the suggested transformation reduces the difficulties that are typical for stiff systems. The efficiency of the approach is shown on test examples.
\end{abstract}

\section{Introduction}

Equations with a small parameter by highest derivative are called singular perturbed equations. They form a class of stiff systems convenient for studying the effectiveness of various numerical methods of integration of stiff systems. New advances in asymptotic theory [2], theory of difference schemes [3] and simplicity of qualitative behavior of solution make possible the detailed analyses of such a system.

We consider the simplest singular perturbed equation of form 4

$$
\varepsilon \frac{d y}{d t}=f(y, t), \quad \varepsilon>0 \text {. }
$$

Consider the case when a degenerated equation, corresponding to the equation (11),

$$
f(x, t)=0
$$

has a unique solution $x=x(t)$ and the value of $\partial f / \partial y$ is negative in the neighborhood of this solution. The latter condition is essential for stability of the solution $x=x(t)$.

\footnotetext{
* Supported by the Russian Foundation for Basic Research, project $N^{o}$ 01-01-00038
} 
The behavior of the equation (1) solution is as follows. If the value of $\varepsilon$ is sufficiently small, the tangents to integral curves are almost parallel to the axis $y$, even for a small deviation from the function $x(t)$. And the smaller is value of $\varepsilon$, the faster the integral curve and solution $x(t)$ of degenerated equation come close.

The situation can be described in the following way. Two intervals of essentially different behavior take place for any integral curve in the domain considered. The duration of the first of them is much smaller than that of the second one. The first interval, where the desired function varies fast, represents the tending of the integral curve to the curve $x(t)$ and is named the boundary layer. In the second interval the derivatives are essentially smaller and the integral curve practically coincide with the curve $x(t)$. The boundary layer always takes place, except for the case when initial condition is a root of degenerate equation, i.e., when $y_{0}=x\left(t_{0}\right)$. The smaller is value of parameter $\varepsilon$, the stronger is the difference of the behavior on the two intervals.

Thus, the solution of the degenerate equation can be used for describing the solution of differential equation (1) outside of the boundary layer. The difficulties of numerical solution of the problems considered result from the fact that the derivative $d y / d t$ increases sharply even for small deviation of initial conditions from the curve $x(t)$ at any of its point.

\section{The Best Continuation Parameter}

We consider the solution of the system of nonlinear algebraic or trancendental equations

$$
F(x)=0, \quad x \in \mathbb{R}^{n+1}, \quad F: \mathbb{R}^{n+1} \rightarrow \mathbb{R}^{n} .
$$

The set of solutions of this equation forms the curve in $\mathbb{R}^{n+1}$.

We will suppose that in equation (2) the unknowns depend of some parameter $\mu$

$$
x=x(\mu)
$$

Then after differentiating equations (2) with respect to $\mu$ we obtain the continuation equations

$$
\bar{J} \frac{d x}{d \mu}=0, \quad \bar{J}=\bar{J}(x)=\frac{\partial F}{\partial x} .
$$

Here $\bar{J}$ coincides with the augmented Jacobi matrix In the vicinity of the point $x$ on the curve of the solutions set we introduce parameter $\mu$ that is measured along the axis determined by identify vector $\alpha=\left(\alpha_{1}, \ldots, \alpha_{n+1}\right)^{T} \in$ $\mathbb{R}^{n+1}, \quad \alpha \alpha=\alpha_{i} \alpha_{i}=1$. Then in this point

$$
d \mu=\alpha d x=\alpha_{i} d x_{i}, \quad i=\overline{1, n+1} .
$$

Here the summation with respect to repeating indexes in mentioned limits is used. 
Choosing the vector $\alpha$ in a different way we can define any continuation parameter.

The continuation equations (4), (5) we write as

$$
\left[\begin{array}{cccc}
\alpha_{1} & \alpha_{2} & \ldots & \alpha_{n+1} \\
F_{1,1} & F_{1,2} & \ldots & F_{1, n+1} \\
\vdots & \vdots & \ddots & \vdots \\
F_{n, 1} & F_{n, 2} & \ldots & F_{n, n+1}
\end{array}\right]\left[\begin{array}{c}
x_{1, \mu} \\
x_{2, \mu} \\
\vdots \\
x_{n+1, \mu}
\end{array}\right]=\left[\begin{array}{c}
1 \\
0 \\
\vdots \\
0
\end{array}\right]
$$

Here $F_{i, j}=\partial F_{i} / \partial x_{j}, \quad x_{i, \mu}=d x_{i} / d \mu$.

We can prove the following assertion [5], 6]

Theorem 1. For the system of linearized equations (6) to become the best conditioned, it is necessary and sufficient to take the length of the curve of the system solution set as the continuation parameter of the nonlinear system (2)

\section{The Best Argument of the Problem}

Consider the Cauchy problem for normal system of ODE's

$$
\frac{d y_{i}}{d t}=f_{i}\left(y_{1}, y_{2}, \ldots, y_{n}, t\right), \quad y_{i}\left(t_{0}\right)=y_{i 0}, \quad i=\overline{1, n}
$$

Assume that the conditions of existence and the uniqueness theorem are fulfilled for this problem.

Let an integral the problem to be given by the relations

$$
\begin{aligned}
& F_{j}\left(y_{1}, \ldots, y_{n}, t\right)=0, \quad j=\overline{1, n}, \\
& F_{j}\left(y_{10}, \ldots, y_{n 0}, t_{0}\right)=0,
\end{aligned}
$$

that define the integral curve of the problem (7) in $(n+1)$ - dimensional Euclidean space $\mathbb{R}^{n+1}:\left\{y_{1}, \ldots, y_{n}, t\right\}$.

The process of finding this curve can be considered as a problem of constructing of the solutions set of the system of equations (8) containing the parameter $\operatorname{argument} t$ for various values of $t$. We will use parametric continuation method to solve this system. Then the problem (7) can be considered as a Cauchy problem for the continuation equations of the system (8) solution with respect to the parameter $t$ when the system is reduced to the normal form. Hence we can look for the best continuation parameter. We will call it the best argument.

We choose the best argument - parameter locally, i.e.,in a small vicinity of each point of the solution set curve - integral curve of the problem (77).

To solve the problem we assume that $y_{i}$ and $t$ are such functions of some argument $\mu$ that at each point of the integral curve of the problem

$$
d \mu=\alpha_{i} d y_{i}+\alpha_{n+1} d t, \quad i=\overline{1, n} .
$$


Here $\alpha_{k}(k=\overline{1, n+1})$ are the components of a unit vector $\alpha=\left(\alpha_{1}, \ldots\right.$, $\left.\alpha_{n+1}\right)^{T}$ determining the direction along which the argument $\mu$ is measured. Note that the summation with respect to repeated subscript $i$ is assumed in the expression (9).

Right - hand side of the equality (9) can be regarded as the scalar product of the vector $\alpha$ and the vector - function differential $\left(d y_{1}, \ldots, d y_{n}, d t\right)^{T}$. Assigning various values to the components of the vector $\alpha$ it is possible to consider all possible continuation parameters of the problem (8), i.e., all arguments of the problem (7).

Since the particular form of equation (8) is unknown the change to the argument $\mu$ can be implemented immediately for the problem (7). Dividing the equality (9) by $d \mu$ after that, we obtain

$$
\begin{gathered}
y_{i, \mu}-f_{i} t,{ }_{\mu}=0 \\
\alpha_{i} y_{i, \mu}+\alpha_{n+1} t,_{\mu}=1 \quad i=\overline{1, n} .
\end{gathered}
$$

If the vector $y=\left(y_{1}, \ldots, y_{n}, t\right)^{T} \in \mathbb{R}^{n+1}$ is introduced, the system (10)can be written in the matrix form

$$
\left[\begin{array}{l}
A \\
\alpha
\end{array}\right] y, \mu=\left[\begin{array}{l}
0 \\
1
\end{array}\right] .
$$

Here the matrix $A$ of size $n \times(n+1)$ has the structure

$$
A=[E f]
$$

where $E$ is the unit matrix of $n$ - th order and $f=\left(f_{1}, \ldots, f_{n}\right)^{T}$ is a vector in $\mathbb{R}^{n}$.

The structure of the system (11) is exactly the same as that of the system (6). Therefore, in according with the Theorem [1, the transition to the normal form of the system (11) is the best conditioned if and only if $\alpha=y, \lambda$, i.e., if the arc length of the curve of the system (8) solution is chosen as the parameter $\mu$. This curve is the integral curve of the problem (7). Thus, the system (11) can be written in the form

$$
\begin{gathered}
y_{i, \lambda} y_{i, \lambda}+t,{ }_{\lambda}^{2}=1 \\
y_{i, \lambda}-f_{i} t, \lambda=0,
\end{gathered}
$$

which can be solved analytically with respect to derivatives. Since the argument does not appear explicitly in the equations we take the initial point of the Cauchy problem (7) as the initial point of $\lambda$. Then we arrive to the following form of the Cauchy problem

$$
\begin{gathered}
\frac{d y_{i}}{d \lambda}=\frac{f_{i}}{\sqrt{1+f_{j} f_{j}}} y_{i}(0)=y_{i 0} \\
\frac{d t}{d \lambda}=\frac{1}{\sqrt{1+f_{j} f_{j}}} t(0)=t_{0} \\
i, j=\overline{1, n} .
\end{gathered}
$$


Below, the argument $\lambda$, which provides the best conditioning to the system of equation (11), will be named as the best argument.

Thus the main result has been proved [7].

Theorem 2. For the Cauchy problem for the normal system of ODE (7) to be transformed to the best argument it is necessary and sufficient that the arc length of the solution curve to be chosen as this argument. In this case the problem (7) is transformed to the problem (13).

The new formulation of the Cauchy problem (13) has a number advantages in comparison with the Cauchy problem (7). First, the right - hand side of each equation (13) does not exceed unit. Moreover, the squared norm of the system right - hand sides is always equal to unit. This removes many of the problems connected with unlimited growth of the right - hand sides of the system (7), and allows to integrate differential equations which have the limiting points at integral curves where the derivatives become infinite. It becomes possible to solve problem with closed integral curves. Also the suggested transformation reduces the difficulties that are typical for stiff systems.

Example 1. Let consider some problems. The first problem, taken from [3], is nonlinear Edsberg's problem

$$
\frac{d y}{d t}=-2 k y^{2}, \quad y(0)=10
$$

It has the exact analytical solution

$$
y(t)=\frac{10}{1+20 k t} .
$$

After $\lambda$ - transformation the problem (14) takes the form

$$
\begin{aligned}
\frac{d y}{d \lambda}=\frac{-2 k y^{2}}{\sqrt{1+4 k^{2} y^{4}}}, & \frac{d t}{d \lambda}=\frac{1}{\sqrt{1+4 k^{2} y^{4}}}, \\
y(0)=10, & t(0)=0 .
\end{aligned}
$$

This problem was integrated by the program PC1 [8] for $k=10^{3}$ with accuracy $10^{-10}$ and initial integration step 0.1 on the interval $t \in[0,1]$. The execution time for this problem was two times less then for the problem (14).

Example 2. As another example, we consider numerical solution of Cauchy problem for Van der Pol's equation 9. If a new variable $t=x / \mu$ is introduced in classical Van der Pol's equation $y^{\prime \prime}-\mu\left(1-y^{2}\right) y^{\prime}+y=0$, the equation take the form $\varepsilon y^{\prime \prime}-\left(1-y^{2}\right) y^{\prime}+y=0$ where $\varepsilon=1 / \mu^{2}$. And Cauchy problem can be formulated in the form

$$
\begin{aligned}
& \frac{d y_{1}}{d t}=y_{2}, \quad \varepsilon \frac{d y_{2}}{d t}=\left(1-y_{1}^{2}\right) y_{2}-y_{1}, \\
& y_{1}(0)=2, \quad y_{2}(0)=-0.66
\end{aligned}
$$


Here the small parameter is $\varepsilon=10^{-6}$.

This is a well - known benchmark for estimation the efficiency of computational programs intended to solve stiff systems.

The program PC1 was used for solving this problem. The accuracy of computations was checked by comparison with "exact results" obtained in [9] and it did not exceed the value of $10^{-3}$. The problem was solved for $t \in[0,0.01]$. The $\lambda$ - transformation reduced execution time tenfold. The number of calculations of the right - hand sides was reduced twenty fold. The integration step of the $\lambda$ - transformed problem was ten times bigger.

Note, that the program PC1 allows to find the solution of $\lambda$ - transformed Van der Pol problem for $t \in[0,2]$ whereas the solution the original problem terminated at $t=0.03$ because of exponent overflow.

Example 3. Consider a initial value problem [3]

$$
\begin{gathered}
\varepsilon \ddot{x}+(1+t) x=2.5(1+t), \\
x(0)=-1, t \in[0,1], \varepsilon=0.003125 .
\end{gathered}
$$

The explicit scheme of Euler method was used for solving system. The program Maple $\mathrm{V}$ was used for solving this problem.

Abbreviations.

$\mathrm{E}$ - for Euler method results

$\mathrm{BP}$ - for results of Euler method for system transformed to the best parameter

$h$ - step size for $t$ or for $\lambda$ (in case of the best parameter)

$\Delta$ - maximal error on $[0,1]$

Table 1 shows results of numerical experiments.

Table 1.

\begin{tabular}{|l|l|l|l|}
\hline method & h & $\Delta$ & $\begin{array}{l}\text { quantity } \\
\text { of steps }\end{array}$ \\
\hline BP & 0.2 & 0.127 & 256 \\
BP & 0.05 & 0.029 & 294 \\
BP & 0.01 & 0.0053 & 586 \\
BP & 0.005 & 0.0025 & 935 \\
E & 0.005 & $\infty$ & 200 \\
E & 0.004 & 1.950 & 250 \\
E & 0.002 & 0.584 & 500 \\
E & 0.001 & 0.239 & 1000 \\
E & 0.0001 & 0.020 & 10000 \\
\hline
\end{tabular}




\section{References}

1. Shalashilin, V.I., Kuznetsov, E.B.: The method of solution continuation with respect to a parameter and the best parametruzation in applied mathematics and mechanics. Editorial URSS, Moscow (1999) (Russian)

2. Vasil'eva, A.B., Butuzov, V.F.: Asymptotic expansions of solutions of singular perturbed equations. Nauka, Moscow (1973) (Russian)

3. Doolan, E.P., Miller, J.J.H., Schilders, W.H.A.: Uniform numerical methods for problems with initial and boundary layers. Boole Press, Dublin (1980)

4. Rakitskii, Yu.V., Ustinov, S.M., Chernorutskii, I.G.: Numerical methods for solving stiff systems. Nauka, Moscow (1979) (Russian)

5. Shalashilin, V.I., Kuznetsov, E.B.: The best parameter in the continuation of a solution. Dokladi Academii Nauk of Russia. Vol. 334.5 (1994) 566-568

6. Kuznetsov, E.B., Shalashilin, V.I.: Cauchy's problem as a problem of continuation with respect to the best parameter. Diff. Urav. V. 306 (1994) 964-971

7. Shalashilin, V.I., Kuznetsov, E.B.: Cauchy problem for nonlinear deformation of systems as a parametric continuation problem. Dokladi Academii Nauk of Russia. Vol. 329. 4 (1993) 426-428

8. Kuznetsov, E.B., Shalashilin, V.I.: Cauchy's problem as a problem of the continuation of a solution with respect to a parameter. Zh. Vychisl. Mat. Mat. Fiz. Vol. 33. 12 (1993) 1792-1805

9. Hairer, E., Wanner, G.: Solving ordinary differential equations II. Stiff and differential - algebraic problems. Springer-Verlag, Berlin (1991) 